\section{SAT0453 DELAYED DENOSUMAB INJECTIONS AND FRACTURES RISK AMONG SUBJECTS WITH OSTEOPOROSIS: A POPULATION-BASED COHORT STUDY}

H. Lyu ${ }^{1,2,3}$, K. Yoshida' ${ }^{2}$ S. S. Zhao ${ }^{4}$, X. García-Albéniz ${ }^{5}$, J. Wei ${ }^{6}$, C. Zeng ${ }^{3}$, S. Tedeschi' ${ }^{2}$, B. Leder ${ }^{7}$, G. Lei ${ }^{3}$, P. Tang ${ }^{1}$, D. Solomon ${ }^{2}{ }^{1}$ National Clinical Research Center for Musculoskeletal Disease, Beijing, China; ${ }^{2}$ Brigham and Women's Hospital, Devision of Rheumatology, Boston, United States of America; ${ }^{3}$ Xiangya Hospital, Central South University, Department of Orthopaedics, Changsha, China; ${ }^{4}$ University of Liverpool, Institute of Ageing and Chronic Disease, Liverpool, United Kingdom; ${ }^{5}$ RTI Health Solutions, Barcelona, Spain; ${ }^{6}$ Xiangya Hospital, Central South University, Health Management Center, Changsha, China; ${ }^{7}$ Massachusetts General Hospital, Endocrine Unit, Department of Medicine, Boston, United States of America

Background: Denosumab is effective for osteoporosis, but discontinuation leads to rapid reversal of its therapeutic effect[1].

Objectives: To estimate the risk for fracture among users of denosumab who delayed subsequent dosages compared with users who received dosages on time.

Methods: Population-based cohort study. We included patients aged over 45 years who initiated denosumab for osteoporosis from UK THIN database, 2010 to 2019. Observational data were used to "emulate a hypothetical trial" $[2,3]$ with three dosing intervals: subsequent denosumab injection 24-28 weeks after prior dose ("on time"), delay by 4-16 weeks ("short delay"), and delay by over 16 weeks ("long delay"). The primary outcome was a composite of all fracture types. Secondary outcomes included major osteoporotic fracture, vertebral fracture, and hip fracture. Results: The rate of composite fracture per 1000 person-years was 58.9 for on-time, 61.7 for short delay, and 85.4 for long delay of subsequent denosumab injections. Compared to on-time injections, short delay had a hazard ratio (HR) for composite fracture $1.03(95 \% \mathrm{Cl} 0.63-1.69)$ and long delay $\mathrm{HR} 1.44(95 \% \mathrm{Cl}$ 0.96-2.17; $\mathrm{p}$ for trend 0.093). For major osteoporotic fractures, short delay had an $\mathrm{HR} 0.94(95 \% \mathrm{Cl} 0.57-1.55)$ and long delay an $\mathrm{HR}$ of $1.69(95 \% \mathrm{Cl} 1.01-2.83$; $\mathrm{p}$ for trend 0.056$)$. For vertebral fractures, short delay had an $\mathrm{HR} 1.48(95 \% \mathrm{Cl}$ 0.58-3.79) and long delay 3.91 (95\% Cl 1.62-9.45; p for trend 0.005).

Conclusion: While delayed subsequent denosumab dosages over 16 weeks was associated with an increased risk of vertebral and major osteoporotic fracture compared to no delay, composite fracture risk was not increased with longer delays.

References:

[1] Cummings SR, Ferrari S, Eastell R, et al. Vertebral Fractures After Discontinuation of Denosumab: A Post Hoc Analysis of the Randomized Placebo-Controlled FREEDOM Trial and Its Extension. J Bone Miner Res, 2017.

[2] Hernán MA. How to estimate the effect of treatment duration on survival outcomes using observational data. BMJ 2018.

[3] Hernán MA, Robins JM. Using Big Data to Emulate a Target Trial When a Randomized Trial Is Not Available. Am J Epidemiol 2016.

Table. Rates and Adjusted Hazard Ratios of Fracture

On-time Short delay Long delay P for linear trend

\section{Composite Fracture}

Rate (per 1000 person-years)

Unadjusted HR $(95 \%)$

Adjusted HR $(95 \% \mathrm{Cl})^{\dagger}$

Major Osteoporotic Fracture

Rate (per 1000 person-years)

Unadjusted HR (95\%)

Adjusted HR $(95 \% \mathrm{Cl})^{\dagger}$

Vertebral Fracture

Rate (per 1000 person-years)

Unadjusted HR (95\%)

Adjusted HR $(95 \% \mathrm{Cl})$

Hip Fracture

Rate (per 1000 person-years)

Unadjusted HR $(95 \%)$

Adjusted $\mathrm{HR}(95 \% \mathrm{Cl})^{\dagger}$

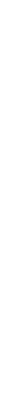

$\begin{array}{cccc}59 & 61.7 & 85.4 & - \\ \text { Ref } & 1.05(0.62,1.76) & 1.45(0.95,2.21) & 0.097 \\ \text { Ref } & 1.03(0.63,1.69) & 1.44(0.96,2.17) & 0.093 \\ & & & \\ 34.8 & 31.9 & 58 & - \\ \text { Ref } & 0.92(0.55,1.53) & 1.67(0.98,2.84) & 0.074 \\ \text { Ref } & 0.94(0.57,1.55) & 1.69(1.01,2.83) & 0.056 \\ & & & \\ 4.9 & 7.3 & 19.4 & - \\ \text { Ref } & 1.47(0.58,3.71) & 3.93(1.59,9.72) & 0.006 \\ \text { Ref } & 1.48(0.58,3.79) & 3.91(1.62,9.45) & 0.005 \\ & & & - \\ 10.2 & 9.6 & 18.3 & - \\ \text { Ref } & 0.94(0.43,2.04) & 1.78(0.80,3.97) & 0.18 \\ \text { Ref } & 0.97(0.44,2.12) & 1.75(0.81,3.79) & 0.173\end{array}$

${ }^{\dagger}$ Adjusted model: adjusted by age, sex, baseline $\mathrm{CCl}$ index, major osteoporotic fracture, oral BP duration (years), 10-year risk of major osteoporotic fracture, prior denosumab doses.

Acknowledgments: We acknowledge Dr. Dani Prieto-Alhambra for kindly providing Read codes.

Disclosure of Interests: Houchen Lyu: None declared, Kazuki Yoshida: None declared, Sizheng Steven Zhao: None declared, Xabier García-Albéniz: None declared, Jie Wei: None declared, Chao Zeng: None declared, Sara Tedeschi: None declared, Benjamin Leder Grant/research support from: Research funding from Amgen, Guanghua Lei: None declared, Peifu Tang: None declared, Daniel Solomon Grant/research support from: Funding from Abbvie and Amgen unrelated to this work

DOI: 10.1136/annrheumdis-2020-eular.5942

\section{SAT0454 EARLY CLINICAL EFFICACY OF ROMOSOZUMAB IN PATIENTS WITH RHEUMATOID ARTHRITIS AND PRIMARY OSTEOPOROSIS}

Y. Kanayama ${ }^{1}$, R. Sugimoto ${ }^{2}{ }^{1}$ Toyota Kosei Hospital, Orthopedic Surgery and Rheumatology, Toyota, Japan; ${ }^{2}$ Toyota Kosei Hospital, Orthopedic Surgery, Toyota, Japan

Background: Romosozumab (ROM), a monoclonal antibody that binds sclerostin, increases bone formation and decreases bone resorption. And although it is a novel therapeutic agent for osteoporosis, which has shown high effects of increasing bone density and inhibiting fragile fracture in overseas clinical trials. However the clinical efficacy in daily clinical practice is unknown.

Objectives: To evaluate the early clinical efficacy of ROM in patients with osteoporosis between rheumatoid arthritis (RA-OP) and primary osteoporosis (P-OP) for 6 months.

Methods: RA patients diagnosed according to the 2010 ACR/EULAR criteria. RA-OP and P-OP patients met at least one of the following criteria were eligible; a bone mineral density $\mathrm{T}$ score of $-2,5$ or less at the lumber spine or total hip and either one or more moderate or severe vertebral fractures or two or more mild vertebral fractures. All patients were initiated ROM from between March and June, 2019. The total number of patients was 15 cases, including 8 RA-OP and 7 P-OP. All patients received continuous ROM therapy more than 6 months. The DMB dose was $210 \mathrm{mg}$ at once every 1 months. In all cases native or activated vitamin $D$ has been used. We reviewed the results for 6 months about the increase and decrease of bone mineral density (BMD) of lumbar spine(LS) and total hip $(\mathrm{TH})$ by DEXA and bone turnover markers, intact $n$-terminal propeptide type I procollagen(PINP) and tartrate-resistant acid phopshatate form 5b(TRACP-5b).

Results: The gender was all female. The mean age was $71.8 \pm 8.7$; disease duration of RA-OP patients was $23.0 \pm 15.1$ years; the body mass index was $19.9 \pm 3.2$ and the FRAX was $32.5 \pm 14.9$. Clinical findings related to RA-OP at baseline were as follows; CRP $0.97 \pm 0.77$; DAS-CRP $3.22 \pm 0.41$; HAQ $1.41 \pm$ 0.94 in RA-OP patients and in the all patients, bone turnover markers and bone mineral density at baseline were as follows; P1NP $72.2 \pm 39.8$; TRACP-5b $539 \pm$ 212; LS-BMD and T-score $0.80 \pm 0.20 \mathrm{~g} / \mathrm{cm}^{2}$ and $-2.75 \pm 1.36$ and TH-BMD 0.55 $\pm 0.07 \mathrm{~g} / \mathrm{cm}^{2}$ and $-3.18 \pm 0.55 \mathrm{~g} / \mathrm{cm}^{2}$. The rate of increased P1NP from baseline to 1,3 and 6 months were each $-96.8 \pm 80.8 \%$ at 1 month, $106.8 \pm 115.6 \%$ at 3 month and $90.7 \pm 115.7 \%$ at 6 month and decreased TRAC- 5 b were -20.4 $\pm 20.6 \%$ at 1 month, $-80.8 \pm 19.6 \%$ at 3 month and $-1.8 \pm 50.8 \%$ at 6 month The rate of increased LS-BMD from baseline to 6 months were $11.0 \pm 8.0 \%$ and TH-BMD were $5.3 \pm 3.8 \%$ (Fig. 1, 2).

Conclusion: Early clinical efficacy of ROM for RA-OP and P-OP was extremely effective and has the high potential to be an important option in the treatment of osteoporosis.

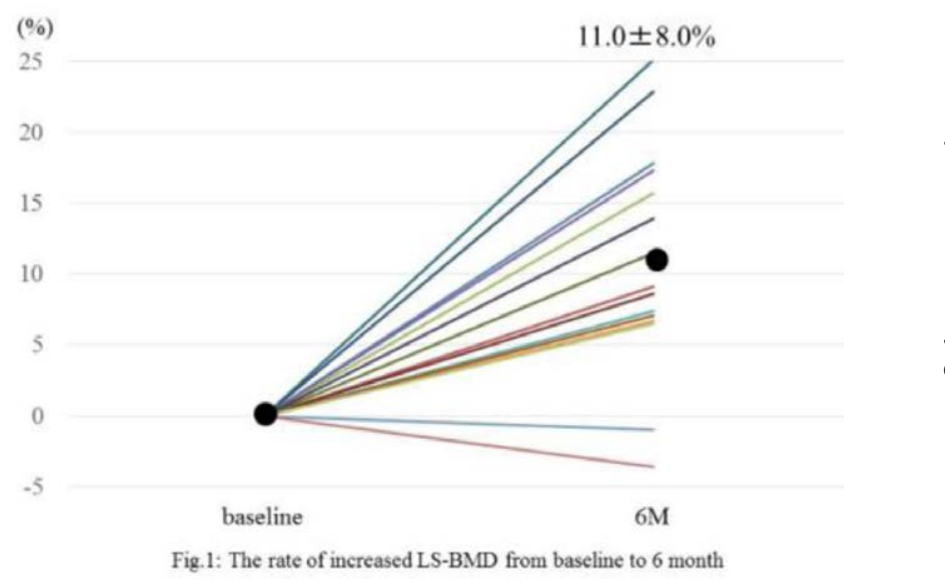

Fig. 1: The rate of increased LS-BMD from baseline to 6 month 


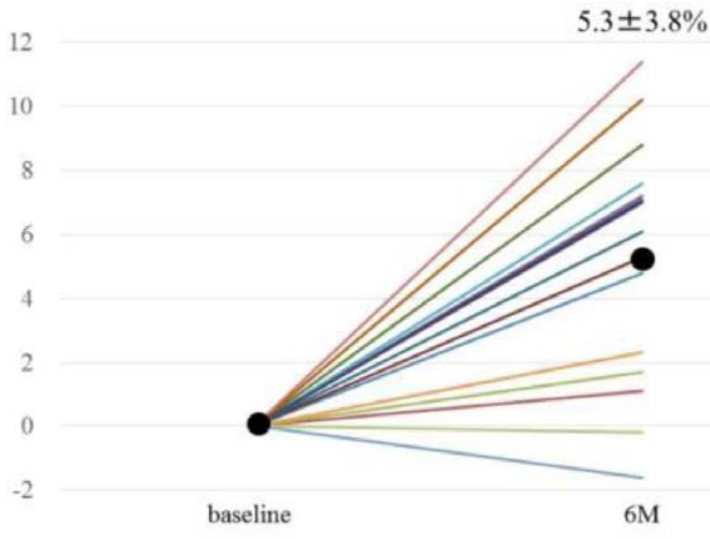

Fig.2: The rate of increased TH-BMD from baseline to 6 month

Disclosure of Interests: None declared

DOI: 10.1136/annrheumdis-2020-eular.5443

\section{SAT0455 RADIOFREQUENCY ECHOGRAPHIC MULTI SPECTROMETRY (REMS) FOR THE IDENTIFICATION OF FRAIL BONES}

G. Adami ${ }^{1}$, G. Arioli ${ }^{2}$, G. Bianchi ${ }^{3}$, M. L. Brandi ${ }^{4}$, C. Caffarelli ${ }^{5}$, L. Cianferotti ${ }^{4}$, G. Girasole ${ }^{3}$, S. Gonnelli ${ }^{5}$, M. Manfedini ${ }^{2}$, M. Muratore $^{6}$, E. Quarta $^{6}$, L. Quarta ${ }^{6}$, D. Gatti' ${ }^{1}{ }^{1}$ University of Verona, Rheumatology Unit, Department of Medicine, Verona, Italy; ${ }^{2}$ Carlo Poma Hospital, ASST-Mantova, Department of Neurosciences and Rehabilitation, Mantova, Italy; ${ }^{3}$ ASL 3 Genovese, SC Rheumatology, Genoa, Italy; ${ }^{4}$ University Hospital of Florence, Department of Surgery and Translational Medicine, Metabolic Bone Diseases Unit, Florence, Italy; ${ }^{5}$ University of Siena, Department of Medicine, Surgery and Neurosciences, Siena, Italy; ${ }^{6}$ Galateo Hospital, ASL-LE, O.U. of Rheumatology, San Cesario di Lecce, Italy

Background: Radiofrequency Echographic Multi Spectrometry (REMS) is the first clinically available approach for direct non-ionizing measurement of bone mineral density (BMD) at lumbar spine (LS) and femoral neck (FN). Available scientific evidences describe BMD estimated by REMS as an accurate parameter for the diagnosis of osteoporosis [1].

Objectives: To investigate the effectiveness of the T-score values provided by REMS scans at FN and LS in the identification of frail patients at risk for osteoporotic fractures and to compare the performance of REMS with the dual-energy X-ray absorptiometry (DXA) one.

Methods: The patients underwent DXA and REMS scans at FN and at LS. Five clusters of fractures occurred during a median 3.5-year follow-up were identified whether involving the upper limb (forearm, elbow, humerus, wrist, hand), lower limb (tibia, ankle, metatarsus), thorax (shoulder blade, shoulder, rib), hip (femur or pelvis bones), or vertebrae. The ability of REMS and DXA T-score values to assess the incidence and site of fractures was evaluated through an analysis of covariance.

Results: Seven hundred twenty-one Caucasian women were enrolled. Ninety-five fractures occurred, in particular 41 at upper limb, 16 at hip, 15 at thorax, 14 at lower limb, 9 at vertebrae. Patients characteristics are reported in table. Considering subcategories of fractured patients, there were not statistically significant differences for age, height, weight and BMI.

In the analysis of covariance including age and BMI as covariates, the difference of T-score values between fractured and non-fractured patients is statistically significant for REMS and DXA at both sites.

Lower FN T-score values were found for patients with fractures at hip or vertebra with respect to non-fractured patients both for REMS and DXA ( $p<0.001)$. Considering LS T-score, lower values were found for patients with fractures at hip, vertebra or upper limb with respect to non-fractured patients both for REMS and DXA $(p<0.001$, Figure).

Conclusion: REMS T-score measured at axial sites is an effective parameter for identification of patients at the risk of incident fragility fractures, in particular occurring at hip, vertebra or upper limb in a population-based sample of female subjects.

References:

[1] Diez-Perez, Aging Clin Exp Res 2019;31(10):1375-89
Table 1. Baseline patient characteristics, expressed as median $\left(25^{\text {th }}-75^{\text {th }}\right.$ percentiles $)$.

\begin{tabular}{lccc}
\hline & $\begin{array}{c}\text { Patients with incident } \\
\text { fragility fracture }\end{array}$ & $\begin{array}{c}\text { Patients without incident } \\
\text { fragility fracture }\end{array}$ & p-value* $^{*}$ \\
\hline Age [years] & $70(60-73)$ & $59(54-64)$ & $<0.001$ \\
Height [cm] & $159(155-164)$ & $160(156-165)$ & 0.08 \\
Weight [kg] & $63(58-70)$ & $62(57-69)$ & 0.42 \\
BMI [kg/m ${ }^{2}$ ] & $24.97(23.13-26.86)$ & $24.24(22.22-26.59)$ & 0.04 \\
FN REMS T-score & $-2.3(-2.8--1.7)$ & $-1.8(-2.3--1.1)$ & $<0.001$ \\
FN DXA T-score & $-2.2(-2.8--1.6)$ & $-1.7(-2.3--1.1)$ & $<0.001$ \\
LS REMS T-score & $-3.0(-3.5--2.0)$ & $-2.0(-2.8--1.0)$ & $<0.001$ \\
LS DXA T-score & $-2.8(-3.4--1.8)$ & $-1.9(-2.7--1.0)$ & $<0.001$ \\
\hline
\end{tabular}

* Wilcoxon ranksum test
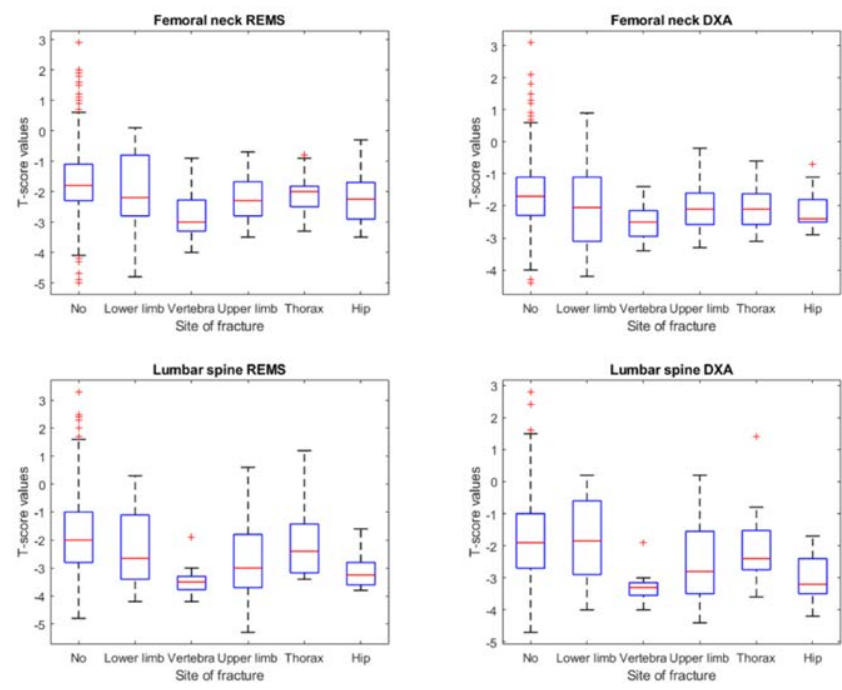

Figure. Boxplot of the distribution of T-score values estimated REMS and DXA at FN and LS among patients without incident fragility fracture and patients with incident fragility fractures at different sites.

Note: G. Adami, G. Arioli ${ }^{\S}$, G. Bianchi ${ }^{\S}$, M.L. Brandi ${ }^{\S}$, C. Caffarelli ${ }^{\S}$, L. Cianferotti ${ }^{\S}$, G. Girasole ${ }^{\S}$, S. Gonnelli ${ }^{\S}$, M. Manfredini $i^{\S}$, M. Muratore ${ }^{\S}$, E. Quarta $^{\S}$, L. Quarta ${ }^{\S}$, D. Gatti $\left.\right|^{\S}$ equal contributors listed in alphabetical order.

Disclosure of Interests: Giovanni Adami: None declared, Giovanni Arioli *: None declared, Gerolamo Bianchi Grant/research support from: Celgene, Consultant of: Amgen, Janssen, Merck Sharp \& Dohme, Novartis, UCB, Speakers bureau: Abbvie, Abiogen, Alfa-Sigma, Amgen, BMS, Celgene, Chiesi, Eli Lilly, GSK, Janssen, Medac, Merck Sharp \& Dohme, Novartis, Pfizer, Roche, Sanofi Genzyme, Servier, UCB, Maria Luisa Brandi: None declared, Carla Caffarelli: None declared, Luisella Cianferotti: None declared, Giuseppe Girasole: None declared, Stefano Gonnelli: None declared, Monica Manfedini: None declared, Maurizio Muratore: None declared, Eugenio Quarta: None declared, Laura Quarta: None declared, Davide Gatti Speakers bureau: Davide Gatti reports personal fees from Abiogen, Amgen, Janssen-Cilag, Mundipharma, outside the submitted work. DOI: 10.1136/annrheumdis-2020-eular.5359

\section{SAT0456 REAL-LIFE RISK OF FRACTURE AND TREATMENT PREVALENCE IN DRUG-INDUCED OSTEOPOROSIS IN ITALY USING A NEW ALGORITHM}

G. Adami ${ }^{1}$, A. Fassio ${ }^{1}$, A. Giollo ${ }^{1}$, G. Orsolini ${ }^{1}$, O. Viapiana ${ }^{1}$, D. Gatti ${ }^{1}$, M. Rossini ${ }^{1}{ }^{1}$ University of Verona, Rheumatology Unit, Verona, Italy

Background: Glucocorticoid-induced osteoporosis and osteoporosis induced by adjuvant hormone therapy for breast cancer are the most common forms of secondary osteoporosis.

Objectives: The exact real-life prevalence of treatment with anti-osteoporotic drugs in women with drug-induced osteoporosis is not known. In the present study, using a new mathematical and computerized algorithm, we investigate the profile of risk of fracture of women with drug-induced osteoporosis and the prevalence of treatment with anti-osteoporotic drugs. 